Synthesis of Natural

Products and

Potential Drugs

\title{
Synthesis of Pyripyropene A
}<smiles>CCC[C@@]1(C)C(=O)C(C)=CCC1C1(C)CO1</smiles>

3 steps from (R)-carvone $\mathrm{dr}=10: 1$

Petersen olefination-epoxide opening

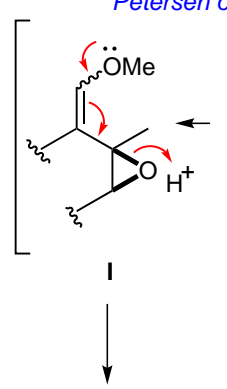
$\underset{\mathrm{Cp}_{2} \mathrm{TiCl}_{2}, \mathrm{Zn}}{\mathrm{THF}, \text { r.t. }}$
$\underset{61 \%}{(21 \% \text { epimer }}$
also isolated $)$

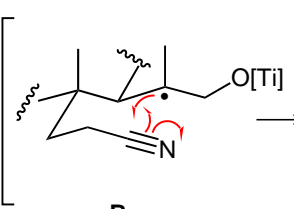

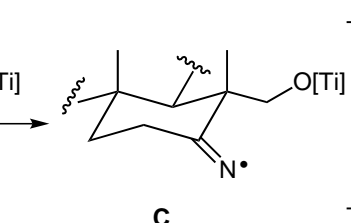<smiles>CC1=CCC2C(CO)C(=O)CCC2(C)C1=O</smiles>

D

\section{Key words}

titanium-mediated radical cyclization

Petersen olefination

pyripyropene A
Significance: Pyripyropene $A$ is a fungal metabolite isolated from Aspergillus fumigatus FO-12892501. It is a potent inhibitor of Acyl-CoA:cholesterol-acyltransferase. Several semi-synthetic derivatives show even greater inhibition.
Comment: Key steps in this synthesis include the titanium-mediated epoxide opening-radical cyclization $(\mathbf{A} \rightarrow \mathbf{D})$ and a Petersen-olefination/epoxide-opening sequence $(\mathbf{F} \rightarrow \mathbf{J})$. A more scalable sequence from $\mathbf{D}$ to $\mathbf{F}$ involving $\mathrm{Me}_{4} \mathrm{NHB}(\mathrm{OAc})_{3}$ as an alternative reducing agent was also reported.

SYNFACTS Contributors: Steven V. Ley, Matthew O'Brien

Dol: 10.1055/s-0031-1289919; Reg-No.: N07111SF 